\title{
Sloshing Dynamics Estimation for Liquid-filled Containers under 2-Dimensional Excitation
}

\author{
Roberto Di Leva $^{1}$, Marco Carricato ${ }^{1}$, Hubert Gattringer ${ }^{2}$, Andreas Müller ${ }^{2}$ \\ ${ }^{1}$ Department of Industrial Engineering \\ University of Bologna \\ ${ }^{2}$ Institute of Robotics \\ Johannes Kepler University Linz \\ Viale del Risorgimento 2, 40136 Bologna, Italy \\ [roberto.dileva, marco.carricato]@unibo.it \\ Altenberger Straße 69, 4040 Linz, Austria \\ [hubert.gattringer, a.mueller]@jku.at
}

\begin{abstract}
Many industrial applications require the displacement of liquid-filled containers on planar paths, by means of linear transport systems or serial robots. The movement of the liquid inside the container, known as sloshing, is usually undesired, so there is the necessity to keep under control the peaks that the liquid free surface exhibits during motion. This paper aims at validating a model for estimating the liquid sloshing height, taking into account 2-dimensional planar motions of a cylindrical container, with accelerations up to $9.5 \mathrm{~m} / \mathrm{s}^{2}$. This model can be exploited for assessment or optimization purposes.

Experiments performed with a robot following three paths, each one of them with different motion profiles, are described. Comparisons between experimental results and model predictions are provided and discussed.
\end{abstract}

Keywords: Sloshing, Prediction model, Validation, Experiments, 2-Dimensional motion.

\section{INTRODUCTION}

The transport of containers filled with liquids finds application in several industrial contexts, e.g. in food\&beverage or pharmaceutical production and packaging lines. Typically, the manipulation of such containers is assigned to linear transport systems or industrial serial robots; in many cases the required motion follows planar curves. The prediction of the liquid movement inside the container, known as sloshing, is important to prevent the liquid from overflowing. Additionally, a reliable sloshing prediction model can be exploited to limit the stirring of the liquid during task execution.

For this purpose, machine-learning methodologies are presented in [1] and [2], where, starting from data collection, predictive algorithms are built to inspect the behavior of discrete liquid particles inside a cylindrical container. This technique, though very powerful, requires experiments to be run in advance, together with a not negligible computational effort.

In [3] the coefficients of the nonlinear sloshing dynamics model presented in [4] are provided to evaluate the sloshing height for 3-dimensional motions, leading to a complex formulation, which may be difficult to use.

A ready-to-use alternative is represented by the development of equivalent discrete mechanical models. The literature considers two main discrete approaches for the modelling of sloshing dynamics inside a container subject to 2-dimensional planar motion [5]: a spherical pendulum and a 2-dof mass-spring-damper system. In the former case, the generalized coordinates describing the system are the angles defining the position of the pendulum mass, whereas, in the latter one, they are the mass displacements from the reference position. Although being intuitive, the use of the angular coordinates of the pendulum mass to assess the sloshing behavior of the liquid (see $[6,7]$ ) lacks physical meaning, above all when the knowledge of the liquid peak height is important. For this reason, in the spherical pendulum model used in [8], [9] and [10], the sloshing height is estimated by means of the tangent functions of the spherical coordinates. However, estimating the 
sloshing height by means of the tangent of the pendulum angles may lead to singularity conditions, when the container acceleration is high, since in this case these angles can approach $90^{\circ}$ and the tangent tend to assume unrealistic high values.

To overcome this drawback, a novel approach, based on the mass-spring-damper model [11], is proposed in [12] for the sloshing-height estimation. This model is validated for 1-dimensional motions and the authors propose the possible extension to planar motions, but no experimental validation is provided. The latter is the objective of this paper, particularly referring to 2-dimensional planar motions of a cylindrical container.

The paper is structured as follows. Section 2 presents the model parameters and the equations of motion (EOMs) of the corresponding generalized coordinates. Section 3 provides the formulation of the sloshing-height estimation. In Section 4, the results from an experimental campaign are described and discussed. Lastly, in Section 5 conclusions are drawn and suggestions for future developments are given.

\section{SLOSHING MODEL}

\subsection{Model Parameters}

We will consider a cylindrical container of radius $R$, filled with a liquid of height $h$ and mass $m_{F}$. A simplified discrete mechanical model can be used to reproduce the liquid-sloshing dynamics. In particular, the mass-spring-damper model comprises a rigid mass $m_{0}$ (whose signed vertical distance from the liquid's center of gravity $G$ is $h_{0}$ ) that moves rigidly with the container, and a series of moving masses $m_{n}$, with each one of them representing the equivalent mass of a sloshing mode (Fig. 1a). Each modal mass $m_{n}$ is restrained by a spring $k_{n}$ and a damper $c_{n}$, and its signed vertical distance from $G$ is $h_{n}$. The model parameters can be determined by imposing a number of equivalence conditions with the original system [5]:

- the overall mass must be the same:

$$
m_{F}=m_{0}+\sum_{n=1}^{\infty} m_{n}
$$

- the height of the center of gravity $G$ must remain the same for small oscillations of the liquid:

$$
m_{0} h_{0}+\sum_{n=1}^{\infty} m_{n} h_{n}=0
$$

- the natural frequency associated with the $n$-th mode must coincide with the one that can be obtained from the continuum model:

$$
\omega_{n}^{2}=\frac{k_{n}}{m_{n}}=g \frac{\xi_{1 n}}{R} \tanh \left(\xi_{1 n} \frac{h}{R}\right)
$$

- the sloshing force acting on the container wall must be the same as the one calculated from the continuum model:

$$
m_{n}=m_{F} \frac{2 R}{\xi_{1 n} h\left(\xi_{1 n}^{2}-1\right)} \tanh \left(\xi_{1 n} \frac{h}{R}\right) .
$$

In Equations (3) and (4), $\xi_{1 n}$ is the root of the derivative of the Bessel function of the first kind with respect to the radial coordinate $r$, for the $1^{\text {st }}$ circumferential mode and the $n$-th radial mode [13], while $g$ is the gravity acceleration. The damping ratio $\zeta_{n}=\frac{c_{n}}{2 \sqrt{k_{n} m_{n}}}$ can be determined by using empirical formulas [5].

For a container under 2-dimensional motion on the horizontal $x y$ plane, the excitation is provided by the container accelerations along the $x$ and $y$ directions, i.e. $\ddot{\mathbf{S}}_{0}=\left[\begin{array}{ll}\ddot{x}_{0} & \ddot{y}_{0}\end{array}\right]^{T}$. The motion of the $n$ th sloshing mass is described by the generalized coordinates $\left(x_{n}, y_{n}\right)$, whose definition is illustrated in Fig. 1b. The latter are then used to compute the liquid sloshing height. 


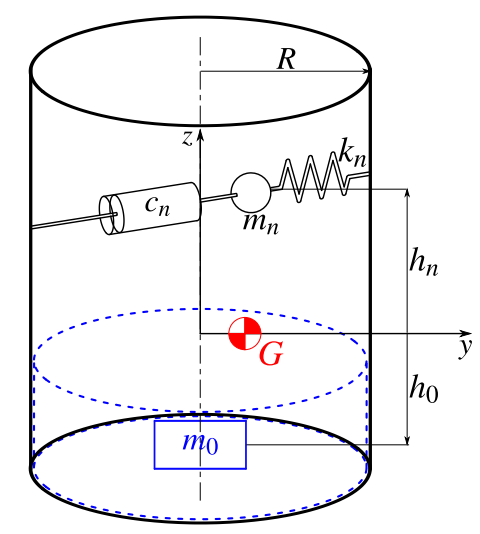

(a) Model Parameters.

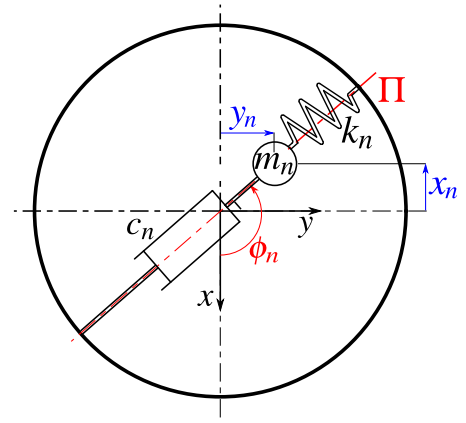

(b) Top view showing the $n$-th generalized coordinates.

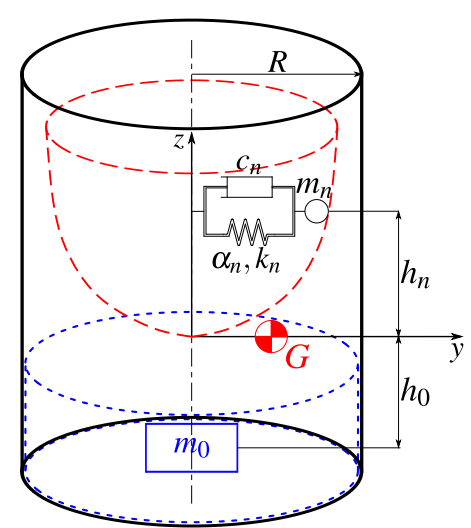

(c) Sloshing mass sliding on a parabolic surface.

Figure 1: Mass-spring-damper model.

\subsection{Equations of Motion}

In general, three dynamic regimes are possible [5]:

- small oscillations in which the liquid free surface remains planar (Fig. 2a);

- relatively-large-amplitude oscillations in which the liquid free surface is no longer planar (Fig. 2b);

- strongly nonlinear motion, where the liquid free surface exhibits instantaneous peaks characterized by swirling shapes.

While the third motion regime will not be object of the present study, the first and second cases can be analyzed by means of a linear mass-spring-damper model ( $\mathrm{L}$ model) and a nonlinear massspring-damper model (NL model), respectively.

The NL model considers the sloshing mass $m_{n}$ as sliding on a parabolic surface, with an attached nonlinear spring of order $w$ (Fig. 1c) [11]. The analytical expression of the parabolic surface allows the writing of the vertical coordinate $z_{n}$ as a function of $x_{n}$ and $y_{n}$, namely:

$$
z_{n}=\frac{C_{n}}{2 R}\left(x_{n}^{2}+y_{n}^{2}\right)
$$

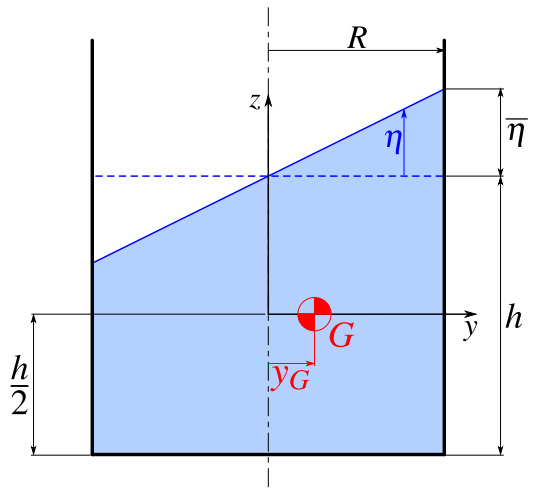

(a) Assumption of planar free surface.

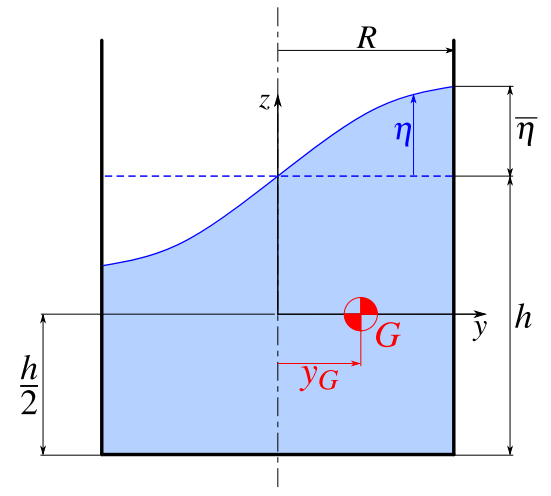

(b) Assumption of nonplanar free surface.

Figure 2: Liquid free-surface shapes. 
where $C_{n}=\omega_{n}^{2} \frac{R}{g}$. The time derivative of Equation (5) yields:

$$
\dot{z}_{n}=\frac{C_{n}}{R}\left(\dot{x}_{n} x_{n}+\dot{y}_{n} y_{n}\right)
$$

The nonlinear spring exerts the forces $\alpha_{n} k_{n} x_{n}^{2 w-1}$ and $\alpha_{n} k_{n} y_{n}^{2 w-1}$, along the $x$ and $y$ direction respectively. In this paper, we choose $w=2$ and $\alpha_{n}=0.58$ [11]. If the radial generalized coordinate $r_{n}=\sqrt{x_{n}^{2}+y_{n}^{2}}$ is introduced, the nonlinear-spring force in the radial direction can be written as $\alpha_{n} k_{n} r_{n}^{2 w-1}$.

The equations of motion (EOMs), describing the time evolution of the generalized coordinates $\left(x_{n}, y_{n}\right)$, can be obtained by means of the Lagrange Equations:

$$
\left\{\begin{array}{c}
\frac{d}{d t}\left(\frac{\partial T}{\partial \dot{x}_{n}}\right)-\frac{\partial T}{\partial x_{n}}+\frac{\partial V}{\partial x_{n}}=-\frac{\partial D}{\partial \dot{x}_{n}} \\
\frac{d}{d t}\left(\frac{\partial T}{\partial \dot{y}_{n}}\right)-\frac{\partial T}{\partial y_{n}}+\frac{\partial V}{\partial y_{n}}=-\frac{\partial D}{\partial \dot{y}_{n}}
\end{array}\right.
$$

where:

- the kinetic energy $T$ of the $n$-th sloshing mass can be computed by taking into account its velocity $\dot{\mathbf{S}}_{n}=\left[\begin{array}{lll}\dot{x}_{n} & \dot{y}_{n} & \dot{z}_{n}\end{array}\right]^{T}$, the container velocity $\dot{\mathbf{S}}_{0}=\left[\begin{array}{ll}\dot{x}_{0} & \dot{y}_{0}\end{array}\right]^{T}$ and by exploiting the Equation (6):

$$
T=\frac{1}{2} m_{n}\left[\left(\dot{x}_{0}+\dot{x}_{n}\right)^{2}+\left(\dot{y}_{0}+\dot{y}_{n}\right)^{2}+\dot{z}_{n}^{2}\right]=\frac{1}{2} m_{n}\left[\left(\dot{x}_{0}+\dot{x}_{n}\right)^{2}+\left(\dot{y}_{0}+\dot{y}_{n}\right)^{2}+\frac{C_{n}^{2}}{R^{2}}\left(\dot{x}_{n} x_{n}+\dot{y}_{n} y_{n}\right)^{2}\right]
$$

- the potential energy $V$ considers the contribution of gravity and nonlinear-spring forces, namely:

$$
V=m_{n} g z_{n}+\int_{0}^{r_{n}} \alpha_{n} k_{n} r_{n}^{2 w-1} d r_{n}=m_{n} g \frac{C_{n}}{2 R}\left(x_{n}^{2}+y_{n}^{2}\right)+\frac{\alpha_{n} k_{n}}{2 w}\left(x_{n}^{2}+y_{n}^{2}\right)^{w}
$$

- the Rayleigh function $D$ accounts for energy dissipation:

$$
D=\frac{1}{2} c_{n}\left(\dot{x}_{n}^{2}+\dot{y}_{n}^{2}+\dot{z}_{n}^{2}\right)=m_{n} \zeta_{n} \omega_{n}\left[\dot{x}_{n}^{2}+\dot{y}_{n}^{2}+\frac{C_{n}^{2}}{R^{2}}\left(\dot{x}_{n} x_{n}+\dot{y}_{n} y_{n}\right)^{2}\right] .
$$

The substitution of Equations (8), (9) and (10) in the system (7) leads to the formulation of two coupled EOMs for the NL model:

$$
\left\{\begin{array}{c}
\ddot{\bar{x}}_{n}+2 \omega_{n} \zeta_{n}\left[\dot{\bar{x}}_{n}+C_{n}^{2}\left(\bar{x}_{n}^{2} \dot{\bar{x}}_{n}+\bar{y}_{n} \dot{\bar{y}}_{n} \bar{x}_{n}\right)\right]+C_{n}^{2}\left(\bar{x}_{n} \dot{\bar{x}}_{n}^{2}+\bar{x}_{n}^{2} \ddot{\bar{x}}_{n}+\bar{x}_{n} \dot{\bar{y}}_{n}^{2}+\bar{x}_{n} \ddot{\bar{y}}_{n} \bar{y}_{n}\right)+ \\
\omega_{n}^{2} \bar{x}_{n}\left[1+\alpha_{n}\left(\bar{x}_{n}^{2}+\bar{y}_{n}^{2}\right)^{w-1}\right]+\frac{\ddot{x}_{0}}{R}=0 \\
\ddot{\bar{y}}_{n}+2 \omega_{n} \zeta_{n}\left[\dot{\bar{y}}_{n}+C_{n}^{2}\left(\bar{y}_{n}^{2} \dot{\bar{y}}_{n}+\bar{x}_{n} \dot{\bar{x}}_{n} \bar{y}_{n}\right)\right]+C_{n}^{2}\left(\bar{y}_{n} \dot{\bar{y}}_{n}^{2}+\bar{y}_{n}^{2} \ddot{\bar{y}}_{n}+\bar{y}_{n} \dot{\bar{x}}_{n}^{2}+\bar{y}_{n} \ddot{\bar{x}}_{n} \bar{x}_{n}\right)+ \\
\omega_{n}^{2} \bar{y}_{n}\left[1+\alpha_{n}\left(\bar{x}_{n}^{2}+\bar{y}_{n}^{2}\right)^{w-1}\right]+\frac{\ddot{y}_{0}}{R}=0
\end{array}\right.
$$

where $\bar{x}_{n}=x_{n} / R, \bar{y}_{n}=y_{n} / R$. As far as the L model is concerned, the linearization of the EOMs in Equation (11) provides two decoupled EOMs in the generalized coordinates $\left(x_{n}, y_{n}\right)$ of the $n$-th mode:

$$
\left\{\begin{array}{l}
\ddot{x}_{n}+2 \zeta_{n} \omega_{n} \dot{x}_{n}+\omega_{n}^{2} x_{n}=-\ddot{x}_{0} \\
\ddot{y}_{n}+2 \zeta_{n} \omega_{n} \dot{y}_{n}+\omega_{n}^{2} y_{n}=-\ddot{y}_{0}
\end{array}\right.
$$


Table 1: SH estimation for a 1-dimensional planar motion.

$\begin{array}{ll}\text { L model } & \bar{\eta}_{n}=\frac{4 h m_{n}}{m_{F} R} y_{n} \\ \text { NL model } & \bar{\eta}_{n}=\frac{\xi_{1 n}^{2} h m_{n}}{m_{F} R} y_{n}\end{array}$

\section{SLOSHING-HEIGHT ESTIMATION}

\subsection{1-Dimensional Motion}

If only a 1-dimensional excitation in the $y$ direction is provided and the phenomenon of rotary sloshing is neglected [5], solely the generalized coordinate $y_{n}$ is different from zero. In such a case, the conservation of the center of gravity $y$-coordinate, between the continuum model and the equivalent model, yields:

$$
y_{G} m_{F}=\sum_{n=1}^{\infty} y_{n} m_{n}+y_{0} m_{0}=\sum_{n=1}^{\infty} y_{n} m_{n}
$$

Considering a cylindrical container of section $S=\pi R^{2}$, filled with a liquid of height $h, y_{G}$ can be computed as:

$$
y_{G}=\frac{1}{S h} \iint_{S} \int_{-\frac{h}{2}}^{\frac{h}{2}+\eta\left(r, \theta, \bar{\eta}_{n}\right)} y d z d S=\frac{1}{\pi R^{2} h} \int_{0}^{R} \int_{0}^{2 \pi} \int_{-\frac{h}{2}}^{\frac{h}{2}+\eta\left(r, \theta, \bar{\eta}_{n}\right)} r^{2} \sin \theta d z d \theta d r,
$$

where the function $\eta\left(r, \theta, \bar{\eta}_{n}\right)$ describes the liquid free-surface shape, $\bar{\eta}_{n}$ is the sloshing height of the $n$-th mode, $(r, \theta)$ are the polar coordinates, with $x=r \cos \theta, y=r \sin \theta, d S=r d \theta d r$. As for the $\mathrm{L}$ model, the function $\eta\left(r, \theta, \bar{\eta}_{n}\right)$ describes a plane (Fig. 2a):

$$
\eta\left(r, \theta, \bar{\eta}_{n}\right)=\sum_{n=1}^{\infty} \bar{\eta}_{n} \frac{r}{R} \sin \theta
$$

whereas, for the NL model, the nonplanar free surface can be described by means of the first-kind Bessel function (Fig. 2b), namely:

$$
\eta\left(r, \theta, \bar{\eta}_{n}\right)=\sum_{n=1}^{\infty} \bar{\eta}_{n} \frac{J_{1}\left(\xi_{1 n} \frac{r}{R}\right)}{J_{1}\left(\xi_{1 n}\right)} \sin \theta
$$

Independently from the adopted function $\eta$, the expression of $y_{G}$ from Equation (14) can be used in Equation (13) to express $\bar{\eta}_{n}$ as a function of the model parameters and the generalized coordinates $\left(x_{n}, y_{n}\right)$, with the latter being obtained by solving the EOMs (see Section 2.2). The L-model assumption of planar surface leads to:

$$
y_{G}=\frac{1}{\pi R^{2} h} \int_{0}^{R} \int_{0}^{2 \pi} \int_{-\frac{h}{2}}^{\frac{h}{2}+\sum \bar{\eta}_{n} \frac{r}{R} \sin \theta} r^{2} \sin \theta d z d \theta d r=\frac{R}{4 h} \sum_{n=1}^{\infty} \bar{\eta}_{n}
$$

Regarding the NL model, by exploiting one of the Bessel function properties, i.e. $\int_{0}^{R} r^{2} J_{1}\left(\xi_{1 n} \frac{r}{R}\right) d r=$ $R^{3} \frac{J_{1}\left(\xi_{1 n}\right)}{\xi_{1 n}^{2}}, y_{G}$ can be evaluated as:

$$
y_{G}=\frac{1}{\pi R^{2} h} \int_{0}^{R} \int_{0}^{2 \pi} \int_{-\frac{h}{2}}^{\frac{h}{2}+\sum \bar{\eta}_{n} \frac{J_{1}\left(\xi_{1 n} \frac{r}{R}\right)}{J_{1}\left(\xi_{1 n}\right)} \sin \theta} r^{2} \sin \theta d z d \theta d r=\frac{R}{h} \sum_{n=1}^{\infty} \frac{\bar{\eta}_{n}}{\xi_{1 n}^{2}}
$$

Inserting the results from Equations (17) and (18) in Equation (13) allows the formulation of the $n$-th sloshing height ( $\mathrm{SH})$ for the L and NL models, respectively, as shown in Table 1. 


\subsection{2-Dimensional Motion}

When accounting for a 2-dimensional excitation, the plane $\Pi$, on which the maximum sloshing height (MSH) occurs, changes its orientation instantaneously, according to a rotation about the $z$-axis by the angle (Fig. 1b):

$$
\phi_{n}=\arctan \left(\frac{y_{n}}{x_{n}}\right)
$$

If the liquid behavior is analyzed on the plane $\Pi$ at every instant, Equation (13) can be extended to the radial coordinate of $G$, remembering that $r_{n}=\sqrt{x_{n}^{2}+y_{n}^{2}}$ :

$$
r_{G} m_{F}=\sum_{n=1}^{\infty} r_{n} m_{n}=\sum_{n=1}^{\infty} m_{n} \sqrt{x_{n}^{2}+y_{n}^{2}}
$$

Equations (17) and (18) can be used to express $r_{G}$ in terms of $\bar{\eta}_{n}$, depending on the adopted model. The approach seen in Section 3.1 allows to write the formulas for the $n$-th maximum sloshing height (MSH) evaluation, both for the L model and the NL model, considering a 2-dimensional motion of the container (Table 2).

\subsection{Remarks}

By looking at Tables 1 and 2, one can point out that, for equal values of the generalized coordinates $\left(x_{n}, y_{n}\right)$, the ratio between $\bar{\eta}_{n}$ obtained from the L model in Equations $(19,23)$ and $\bar{\eta}_{n}$ from the NL model in Equations $(20,24)$ is always $4 / \xi_{1 n}^{2}$. If only the $1^{\text {st }}$ mode is considered, this ratio is equal to $4 / \xi_{11}^{2} \approx 1.18$ and shows that the assumption of planar free surface always overestimates the sloshing height compared to the assumption of a nonplanar free surface. Furthermore, while in Equations $(19,20), \bar{\eta}_{n}$ has the same sign of $y_{n}$, in Equations $(23,24) \bar{\eta}_{n}$ is always positive. This means that Equations $(19,20)$ express the trend of the sloshing height on only one side of the container (on the other side, the sloshing height is estimated as the opposite of $\bar{\eta}_{n}$ ): in this case, we will simply talk about sloshing height (SH). Conversely, in Equations $(23,24), \bar{\eta}_{n}$ indicates the maximum peak that occurs on the container wall (on a plane oriented as described in Equation (21)): in this case, we will use the expression maximum sloshing height (MSH).

\section{EXPERIMENTAL RESULTS}

The experimental setup considers a cylindrical container with radius $R=50 \mathrm{~mm}$ and a liquid static height $h=70 \mathrm{~mm}$. The employed liquid is water, which is colored by adding dark brown powder, in order to obtain a better contrast for the image processing analysis. Motions are performed by an industrial robot (Stäubli RX130L) and recorded by a GoPro Hero3 camera. The trajectories are planned so that the robot follows three geometrical paths (Fig. 3), each one of them with different motion profiles, characterized by increasing container accelerations:

- a back-and-forth linear path (indicated as l-motion);

- an eight-shaped path (e-motion);

- a circular path, performed twice in succession (c-motion).

Table 2: MSH estimation for a 2-dimensional planar motion.

$$
\begin{array}{ll}
\text { L model } & \bar{\eta}_{n}=\frac{4 h m_{n}}{m_{F} R} \sqrt{x_{n}^{2}+y_{n}^{2}} \\
\text { NL model } & \bar{\eta}_{n}=\frac{\xi_{1 n}^{2} h m_{n}}{m_{F} R} \sqrt{x_{n}^{2}+y_{n}^{2}}
\end{array}
$$




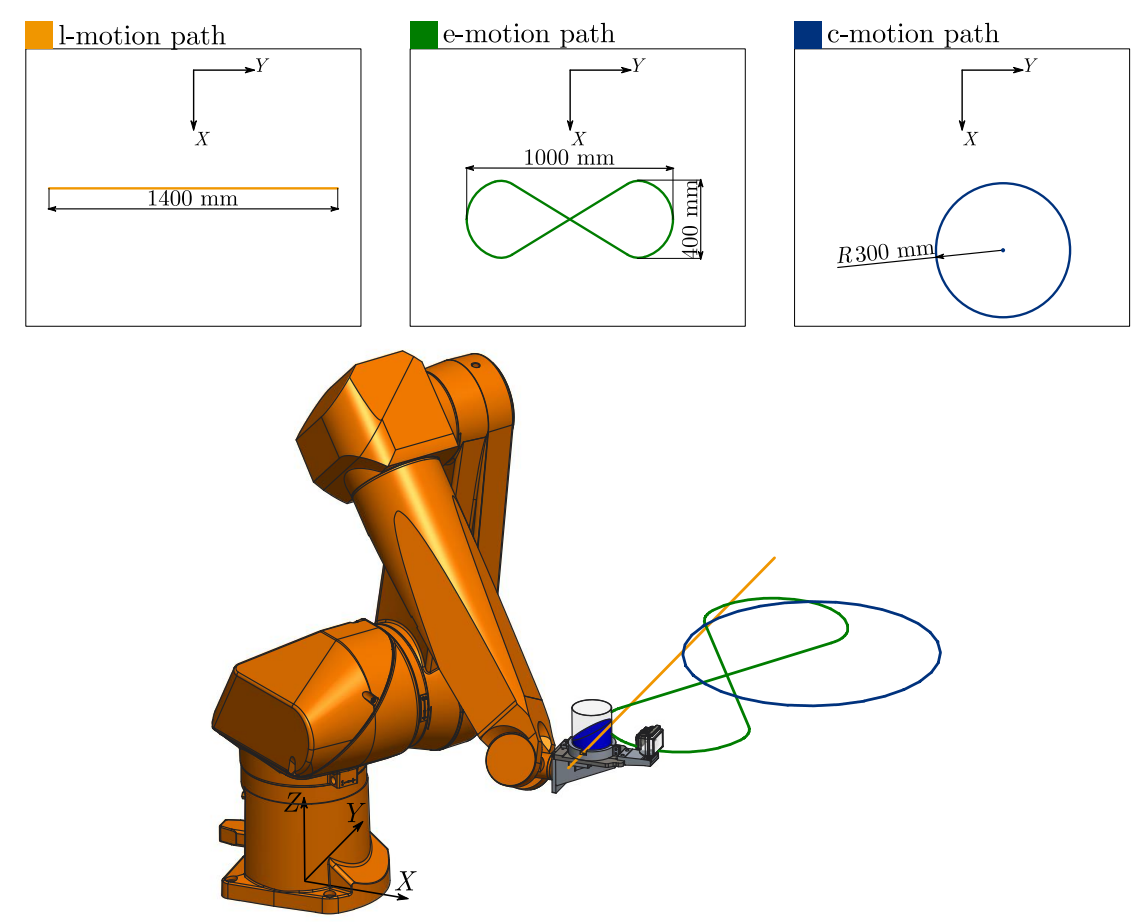

Figure 3: The three paths followed by the robot during experimental validation.

In Fig. 4, the trends of the path parameter second time derivative ${ }^{1} \ddot{\sigma}$ are illustrated: for every path, all three motion profiles are showed. Note that the legend refers to the maximum of the container acceleration norm $\left\|\ddot{\mathbf{S}}_{0}\right\|_{\max }$ reached during the corresponding motion.

In Fig. 5, the L and NL model predictions are compared with the results from the experimental motions, only considering the $1^{\text {st }}$ sloshing mode. A good adherence between the experiments and the models can be appreciated for the 1-dimensional motions (Fig. 5a, 5b, 5c), and the tracking remains reliable also for 2-dimensional motions, especially when considering lower values of $\left\|\ddot{\mathbf{S}}_{0}\right\|_{\max }$ (Fig. 5d, 5g). As the value of the 2-dimensional excitation $\ddot{\mathbf{S}}_{0}$ is increased, the model predictions still capture the trend of the real liquid MSH, although they seem to lose accuracy in correspondence of the peaks reached by the liquid (Fig. 5e, 5f, 5h, 5i). This can be eventually attributable to two reasons:

- the high dynamics given to the container causes a regime of strongly nonlinear motions,

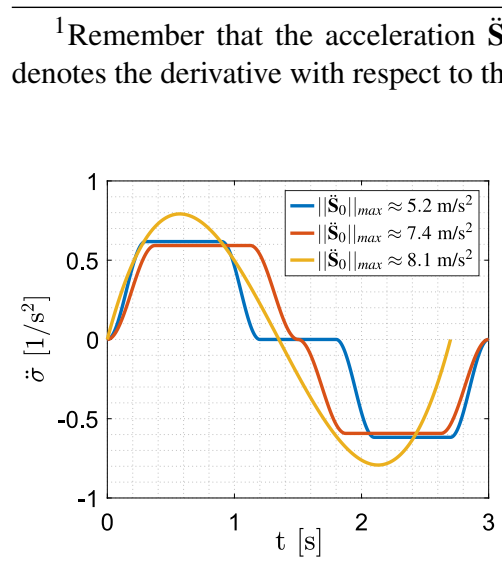

(a) 1-motion.

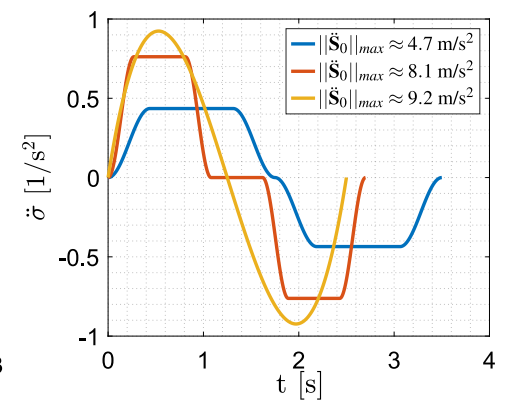

(b) e-motion.

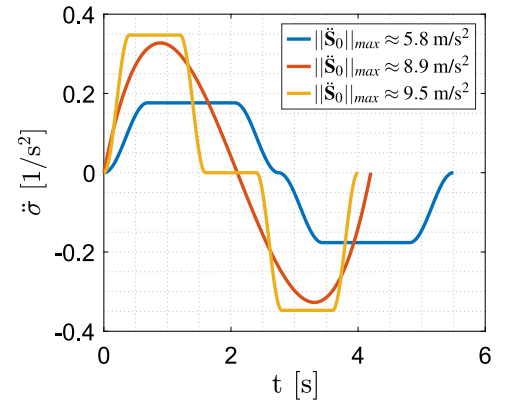

(c) c-motion.

Figure 4: The three motion profiles performed per each path. 


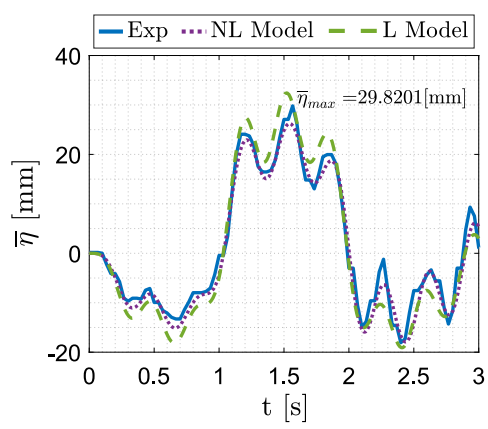

(a) 1-motion: $\left\|\ddot{\mathbf{S}}_{0}\right\|_{\max } \approx 5.2 \mathrm{~m} / \mathrm{s}^{2}$.

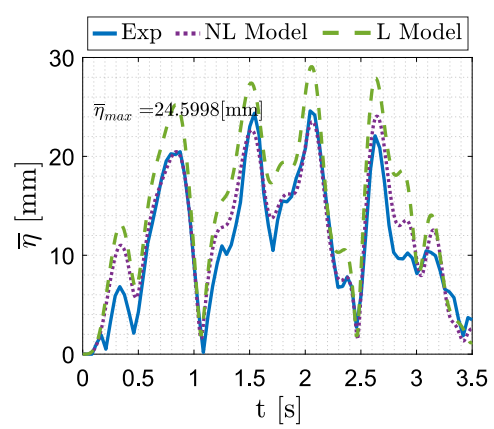

(d) e-motion: $\left\|\ddot{\mathbf{S}}_{0}\right\|_{\max } \approx 4.7 \mathrm{~m} / \mathrm{s}^{2}$.

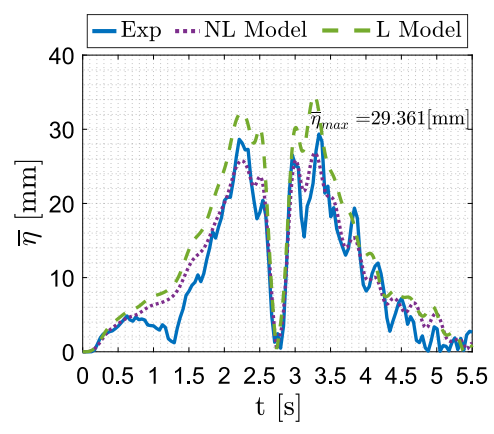

(g) c-motion: $\left\|\ddot{\mathbf{S}}_{0}\right\|_{\max } \approx 5.8 \mathrm{~m} / \mathrm{s}^{2}$.

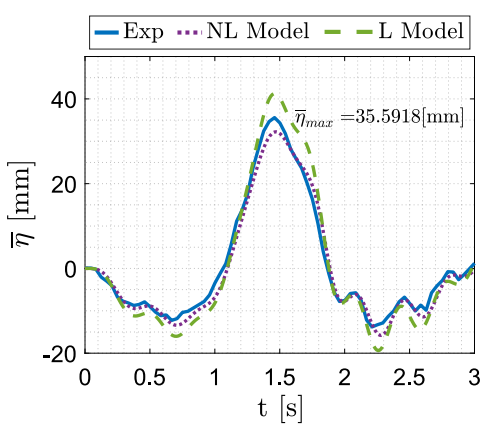

(b) 1-motion: $\left\|\ddot{\mathbf{S}}_{0}\right\|_{\max } \approx 7.4 \mathrm{~m} / \mathrm{s}^{2}$

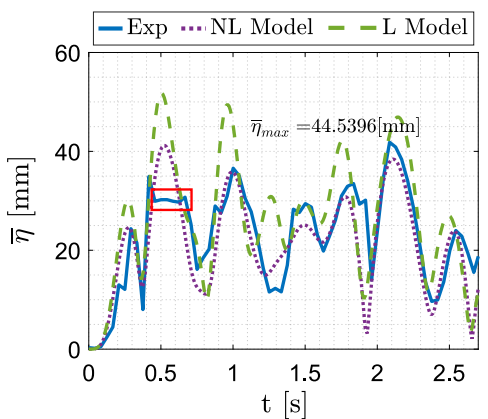

(e) e-motion: $\left\|\ddot{\mathbf{S}}_{0}\right\|_{\max } \approx 8.1 \mathrm{~m} / \mathrm{s}^{2}$.

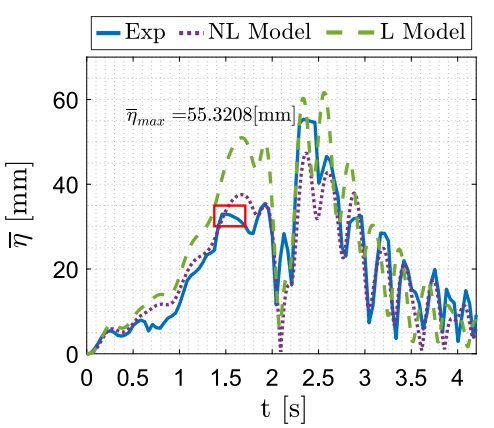

(h) c-motion: $\left\|\ddot{\mathbf{S}}_{0}\right\|_{\max } \approx 8.9 \mathrm{~m} / \mathrm{s}^{2}$.

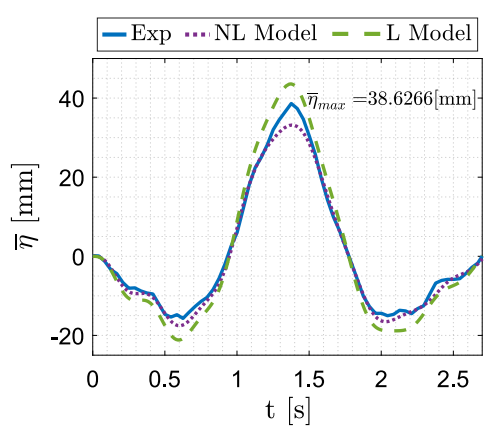

(c) 1-motion: $\left\|\ddot{\mathbf{S}}_{0}\right\|_{\max } \approx 8.1 \mathrm{~m} / \mathrm{s}^{2}$.

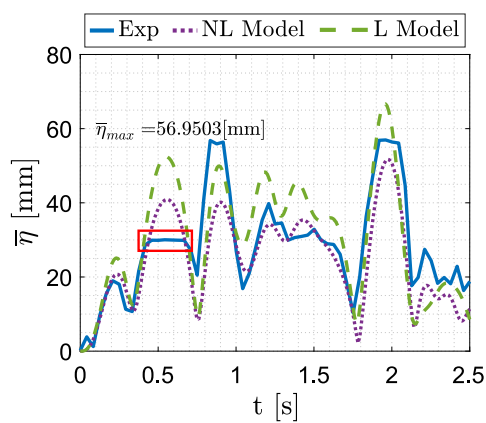

(f) e-motion: $\left\|\ddot{\mathbf{S}}_{0}\right\|_{\max } \approx 9.2 \mathrm{~m} / \mathrm{s}^{2}$.

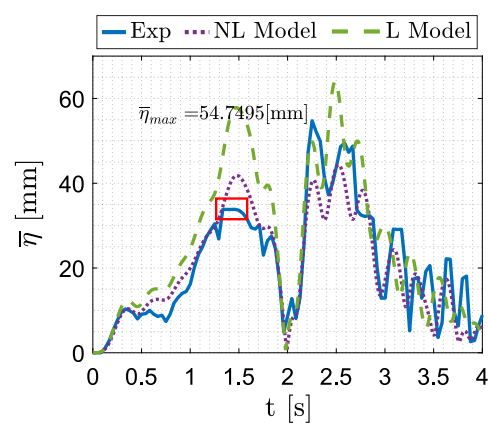

(i) c-motion: $\left\|\ddot{\mathbf{S}}_{0}\right\|_{\max } \approx 9.5 \mathrm{~m} / \mathrm{s}^{2}$.

Figure 5: Comparison between the proposed models and the experimental results.

where the liquid free surface loses the assumed shape and shows instantaneous swirly peaks, as illustrated in Fig. 6a;

- the height of the frames that are employed for the image processing analysis, grants a greater field of view when the liquid peak occurs on the rear wall of the container (Fig. 6b), whereas, for a peak on the front wall (Fig. 6c), the value of the real MSH is saturated by the frame upper limit.

For instance, the latter reason explains the discrepancy between the experiments and the prediction models in the red areas that are highlighted in Fig. 5e, 5f, 5h, $5 \mathrm{i}$.

Table 3 summarizes the obtained results by reporting the accuracy index

$$
\% \varepsilon_{\text {mod }}=\frac{\bar{\eta}_{\text {max }, \text { mod }}-\bar{\eta}_{\text {max }, \text { exp }}}{\bar{\eta}_{\text {max }, \text { exp }}} \times 100
$$

where $\bmod$ and $\exp$ denote the adopted model (L or NL) and the experimental results, respectively. For all motions, $\left|\% \varepsilon_{L}\right|$ is always below the $18 \%$, and $\left|\% \varepsilon_{N L}\right|$ never exceeds $19 \%$, with the NL model granting a better tracking during the whole time period. Furthermore, the positive sign of 


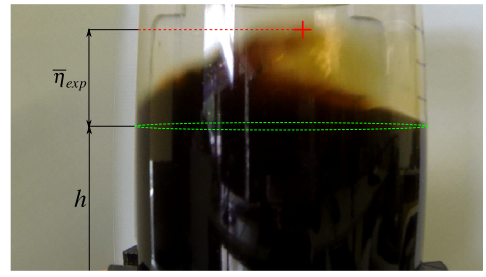

(a) Swirly peak.

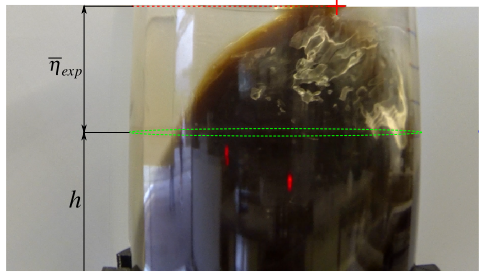

(b) Back peak.

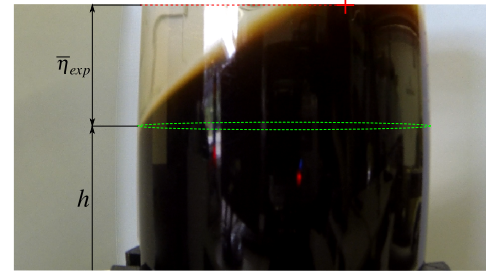

(c) Front peak.

Figure 6: Snapshots from the recorded videos, showing the different peaks reached by the liquid.

Table 3: Accuracy index both for the L model and the NL model.

\begin{tabular}{|c|c|c|c|}
\hline \multirow[t]{3}{*}{ 1-motion } & $\left\|\ddot{\mathbf{S}}_{0}\right\|_{\max } \approx 5.2 \mathrm{~m} / \mathrm{s}^{2}$ & $\left\|\ddot{\mathbf{S}}_{0}\right\|_{\max } \approx 7.4 \mathrm{~m} / \mathrm{s}^{2}$ & $\left\|\ddot{\mathbf{S}}_{0}\right\|_{\max } \approx 8.1 \mathrm{~m} / \mathrm{s}^{2}$ \\
\hline & $\% \varepsilon_{N L}=-11.9 \%$ & $\% \varepsilon_{N L}=-9.5 \%$ & $\% \varepsilon_{N L}=-14.1 \%$ \\
\hline & $\% \varepsilon_{L}=8.8 \%$ & $\% \varepsilon_{L}=15.8 \%$ & $\% \varepsilon_{L}=12.8 \%$ \\
\hline \multirow[t]{3}{*}{ e-motion } & $\left\|\ddot{\mathbf{S}}_{0}\right\|_{\max } \approx 4.7 \mathrm{~m} / \mathrm{s}^{2}$ & $\left\|\ddot{\mathbf{S}}_{0}\right\|_{\max } \approx 8.1 \mathrm{~m} / \mathrm{s}^{2}$ & $\left\|\ddot{\mathbf{S}}_{0}\right\|_{\max } \approx 9.2 \mathrm{~m} / \mathrm{s}^{2}$ \\
\hline & $\% \varepsilon_{N L}=-2.1 \%$ & $\% \varepsilon_{N L}=-7.5 \%$ & $\% \varepsilon_{N L}=-9.2 \%$ \\
\hline & $\% \varepsilon_{L}=18.3 \%$ & $\% \varepsilon_{L}=16.2 \%$ & $\% \varepsilon_{L}=17.1 \%$ \\
\hline \multirow[t]{3}{*}{ c-motion } & $\left\|\ddot{\mathbf{S}}_{0}\right\|_{\max } \approx 5.8 \mathrm{~m} / \mathrm{s}^{2}$ & $\left\|\ddot{\mathbf{S}}_{0}\right\|_{\max } \approx 8.9 \mathrm{~m} / \mathrm{s}^{2}$ & $\left\|\ddot{\mathbf{S}}_{0}\right\|_{\max } \approx 9.5 \mathrm{~m} / \mathrm{s}^{2}$ \\
\hline & $\% \varepsilon_{N L}=-8.7 \%$ & $\% \varepsilon_{N L}=-14.3 \%$ & $\% \varepsilon_{N L}=-19 \%$ \\
\hline & $\% \varepsilon_{L}=17.6 \%$ & $\% \varepsilon_{L}=11.6 \%$ & $\% \varepsilon_{L}=16.9 \%$ \\
\hline
\end{tabular}

$\% \varepsilon_{L}$ proves that the L model always overestimates the real $\mathrm{SH}$ and MSH peaks, as expected (see Section 3.3), hence providing a more conservative estimation.

\section{CONCLUSIONS AND FUTURE WORK}

A novel technique for the sloshing-height estimation of a liquid inside a cylindrical container subject to 2-dimensional planar motions was proposed, extending what was presented in [12]. The technique is based on simple discrete mechanical models, rather than machine-learning or complex fluidodynamical methodologies, thus granting a reliable and easy-to-compute estimation.

Experiments, considering three container paths performed by an industrial robot with different motion profiles, were presented and the relative results were discussed. An accuracy index was used to prove the effectiveness of the estimation, even for high values of the container acceleration (up to $9.5 \mathrm{~m} / \mathrm{s}^{2}$ ).

Future work will address the use of the proposed sloshing-height estimation for square-section containers, adapting the formulation that was presented in Section 3. Moreover, the extension of the technique to 3 -dimensional motions will be investigated. In [6, 7] the additional term $\ddot{z}_{0}$ is treated as a quantity only affecting the $n$-th natural frequency $\omega_{n}$. This approach will be transferred to the $\mathrm{L}$ and NL models and verified by experimental tests.

\section{REFERENCES}

[1] B. Moya, D. Gonzalez, I. Alfaro, F. Chinesta and E. Cueto. Learning Slosh Dynamics by Means of Data. Computational Mechanics, 64(2):511-523, 2019.

[2] B. Moya, I. Alfaro, D. Gonzalez, F. Chinesta and E. Cueto. Physically Sound, Self-learning Digital Twins for Sloshing Fluids. PLOS ONE, 15(6):1-16, 2020. 
[3] J. Huang and X. Zhao. Control of Three-Dimensional Nonlinear Slosh in Moving Rectangular Containers. Journal of Dynamic Systems, Measurement, and Control, 140(8), 2018.

[4] Odd M. Faltinsen, Olav F. Rognebakke and Alexander N. Timokha. Resonant threedimensional nonlinear sloshing in a square-base basin. Journal of Fluid Mechanics, 487:1-42, 2003.

[5] Raouf A. Ibrahim. Liquid Sloshing Dynamics: Theory and Applications. Cambridge University Press, 2005.

[6] W. Aribowo, T. Yamashita, and K. Terashima. Integrated Trajectory Planning and Sloshing Suppression for Three-dimensional Motion of Liquid Container Transfer Robot Arm. Journal of Robotics, 2015.

[7] J. Reinhold, M. Amersdorfer, and T. Meurer. A Dynamic Optimization Approach for Sloshing Free Transport of Liquid Filled Containers using an Industrial Robot. In 2019 IEEE/RSJ International Conference on Intelligent Robots and Systems (IROS), pages 2336-2341, 2019.

[8] L. Moriello, L. Biagiotti, C. Melchiorri, and A. Paoli. Control of Liquid Handling Robotic Systems: A Feed-forward Approach to Suppress Sloshing. In 2017 IEEE International Conference on Robotics and Automation (ICRA), pages 4286-4291, 2017.

[9] L. Moriello, L. Biagiotti, C. Melchiorri, and A. Paoli. Manipulating Liquids with Robots: A Sloshing-free Solution. Control Engineering Practice, 78:129-141, 2018.

[10] L. Biagiotti, D. Chiaravalli, L. Moriello, and C. Melchiorri. A Plug-In Feed-Forward Control for Sloshing Suppression in Robotic Teleoperation Tasks. In 2018 IEEE/RSJ International Conference on Intelligent Robots and Systems (IROS), pages 5855-5860, 2018.

[11] H. Bauer. Nonlinear Mechanical Model for the Description of Propellant Sloshing. AIAA Journal, 4:1662-1668, 1966.

[12] L. Guagliumi, A. Berti, E. Monti, and M. Carricato. A Simple Model-based Method for Sloshing Estimation in Liquid Transfer in Automatic Machines. IEEE Access, 9:129347129357, 2021.

[13] H. Bauer. Tables of Zeros of Cross Product Bessel Functions. Mathematics of Computation, 18(85):128-135, 1964. 